Background: Angiosarcoma is a rare malignant neoplasm that usually involves the skin and subcutaneous tissue. Angiosarcoma of the gastrointestinal tract is extremely uncommon and may demonstrate multicentric epithelioid morphology.

Design: We report a case of a 51-year-old male patient with disseminated epithelioid angiosarcoma (DEA) involving both small and large intestine, who presented with anemia, jaundice, melena and active lower gastrointestinal (GI) bleed since two months. The patient also gave a history of hip surgery two months back and long term analgesic abuse. Endoscopic examination of upper GIT and colon revealed erosions and actively bleeding ulcers respectively. Computed tomography (CT) arteriography revealed active contrast extravasation into proximal ileum. Emergency exploratory laparotomy revealed multiple ulcers in right colon and multiple polyps with erosions in the ileum. Surgical resection-anastomosis of the lesions were done. Histopathological and immunohistochemical assessment revealed a diagnosis of DEA.

Results: On post operative day three the patient developed disseminated intravascular coagulopathy and could not be revived.

Conclusions: Intestinal angiosarcoma is a diagnostic challenge because it is rare and presents with non specific symptoms. A high index of suspicion is warranted in patients presenting with multiple polyps and active GI bleeding. The appropriate classification and treatment of angiosarcomas is important as they are clinically aggressive tumors. Angiosarcomas of the gastrointestinal tract commonly display epithelioid cytomorphology, are often positive for cytokeratins and only show subtle signs of vascular differentiation, creating potential diagnostic confusion with primary or metastatic carcinoma. Immunohistochemistry with vascular markers, CK20, and S-100 protein may be helpful in differentiating angiosarcoma from carcinoma and melanoma.

\section{A MITOTICALLY ACTIVE SOMATIC SOFT TISSUE TUMOUR OF UNCERTAIN POTENTIAL IN A 16 YEAR OLD MALE}

\author{
Joshua P. Hopp \\ Anatomical Pathology Department, Pathology West, Wagga \\ Wagga, NSW, Australia
}

Background: A 16 year old male underwent core biopsy of a painless, deep-seated L pectoralis major lesion, which was reported as possible myofibroma. Complete excision was recommended.

Pathology: Macroscopically the lesion weighed $8.9 \mathrm{~g}$ as was irregular and thinly encapsulated and had a homogenous, firm and white cut surface. Microscopically, the tumour consisted of bundles of spindle cells consistent with smooth muscle, with mild nuclear atypia but no evidence of infiltrative growth. Mitoses were $2 /$ high power $(\times 40)$ field. Immunohistochemically, there was strong positive staining for desmin, SMA and actin, confirming smooth muscle histogenesis. The Ki67 index was $10 \%$. Melanocytic markers were negative and there was patchy positivity for pancytokeratin.

Discussion: Deep soft tissue/somatic leiomyomas in adolescent patients are exceedingly rare. A vascular smooth muscle origin has been postulated. Smooth muscle tumours in childhood with features like those encountered in this case have been considered as leiomyosarcomas by some authorities. However, in such cases no patient developed metastasis, raising the question of whether they are truly malignant or mitotically active leiomyomas, similar to those found in the adult female reproductive tract. Based on the absence of major cytological atypia and necrosis, and the only mildly increased mitotic rate and proliferation index, we favour a diagnosis of mitotically active smooth muscle tumour of uncertain malignancy potential for the current case. The diagnosis was reached in consultation with an expert in softtissue tumour pathology. At three year follow up, the patient has experienced no confirmed recurrence.

\section{ELEVATED BETA-HCG IN A NON-PREGNANT WOMAN? ALSO CONSIDER LUNG ADENOCARCINOMA: A CASE REPORT}

Zaid Househ $^{1}$, Kyaw Lynnhtun ${ }^{1}$, Amit Kapur ${ }^{2}$, Philip Clingan ${ }^{2}$

${ }^{1}$ Anatomical Pathology Department, and ${ }^{2}$ Department of Medical Oncology, The Wollongong Hospital, Wollongong, NSW, Australia

An elevated beta-hCG level (Beta subunit of human chorionic gonadotropin) in a non-pregnant woman raises the suspicion of a germ cell tumour especially choriocarcinoma. Paraneoplastic syndrome involving beta-hCG secretion in other malignancies can be easily overlooked. We encountered a case in which the patient was found to have an elevated beta-hCG level and the clinical diagnosis was further confounded by the finding of a pelvic mass. A 50-year-old post-menopausal woman with a history of smoking was found to have a high beta-hCG level (128 IU/L) during investigation following motor vehicle accident. Pelvic ultrasound at that time showed no evidence of intrauterine or ectopic pregnancy and no tumour. CT scan of the chest showed a $3 \mathrm{~mm}$ nodule in the right middle lobe. No other tumour markers were elevated. Within three months, serum beta-HCG level rose up to $1206 \mathrm{IU} / \mathrm{L}$, and CT scan and PET scan showed multiple lesions in lungs, mediastinum, liver, left pelvis, bone and right adrenal gland.

Subsequent biopsy of the left pelvic mass showed an adenocarcinoma with DPAS-positive cytoplasmic vacuoles and positive expression of pan-cytokeratin, CK7 and TTF-1. Beta-HCG was diffusely positive in the malignant cells as well as in background stroma. PLAP, CD117, CD30, AFP, CK20, CDX-2, PAX-8, ER, PR, P63 and WT-1 were negative. Overall features were consistent with a metastasizing pulmonary adenocarcinoma with paraneoplastic beta-hCG secretion. The possibility of sampling limitation of a somatic malignancy arising from mixed germ cell tumour was considered but there were no pelvic tumour identified in initial presentation and no evidence of germ cell tumour in the biopsy sample.

\section{PSEUDOANGIOSARCOMA OF THYROID - A WORRISOME HISTOLOGICAL ALTERATION FOLLOWING FINE NEEDLE ASPIRATION (WHAFFT)}

K. Iyengar ${ }^{1}$, C. Wong ${ }^{2}$, F. Fiumara ${ }^{3}$, M. Singh ${ }^{1,4}$

${ }_{1}^{1}$ Department of Anatomical Pathology, Pathology Queensland,

${ }^{2}$ Department of Breast and Endocrine Surgery, ${ }^{3}$ Department of Nuclear Medicine and Queensland Specialised PET Service, 
Royal Brisbane and Women's Hospital, and ${ }^{4}$ Discipline of Molecular and Cellular pathology, The University of Queensland, Australia

Fine needle aspiration cytology is often the first or second line of investigation in the presence of enlarged thyroid gland or thyroid nodule, with a high sensitivity and specificity for neoplasms. Changes described as WHAFFT have been identified in subsequent histopathological examination. These range from nuclear pseudoinclusions mimicking papillary carcinoma, pseudoinvasion of thyroid capsule, infarction and vascular proliferation mimicking haemangiomas or papillary endothelial hyperplasia. Awareness of these changes may help to alleviate some of the stresses of diagnostic histopathological work. We present a recent case in which a similar change was identified in a 70 year old male with a $6 \mathrm{~mm}$ diameter nodule in the right lobe of thyroid. A fine needle aspiration cytology was reported as atypical follicular cells (Bethesda Category 3). Six weeks later, a right hemithyroidectomy was performed which showed a papillary microcarcinoma. A nodule of high vascularity and a needle tract were noted in the adjacent tissue. The differential diagnosis of such a nodule and ancillary tests aided in arriving at a final diagnosis is discussed.

\section{TRIPLICATION OF THE APPENDIX: A CASE REPORT}

Annie H. Jo, Brooke Beardsley

Canterbury Health Laboratories, Christchurch, New Zealand

Introduction: Duplication of the appendix has an incidence of $0.004 \%$. From 1892, cases have been reported in the literature, and have led to the Cave-Wallbridge classification system. Appendiceal triplications are rarer still, with only two cases of appendiceal triplication having been described in the literature. This is the first reported case of a single appendix with triplication of the lumen, each, at least focally, with its own separate muscular layer.

Case: A male aged 21 presented with abdominal pain. Examination revealed tenderness and guarding in the right iliac fossa. White count was normal, with CRP 34. Laparoscopic was converted to open appendicectomy as the inflamed appendix was adherent to terminal ileum and abdominal wall.

Pathology: Macroscopically, the base of the appendix had one lumen. This divided into a second adjacent lumen which split again resulting in three distinct lumens spanning a few transverse sections. The lumens then merged back into two, then into a single lumen at the tip.

Microscopically, three separate lumens were confirmed. Focally, each lumen had its own mucosa, submucosa, and muscularis propria with surrounding fibrofatty tissue. Acute appendicitis was also confirmed.

Discussion: These three cases of triplication could expand upon the Cave-Wallbridge classification for appendiceal duplication. The 1968 case describes a Type B triplication with three separate appendices arising from a single caecum. Our case and the 2006 case are variations of Type A. Our case has three lumens, focally with separate muscular layers, which extend partially through the length of the appendix. The other case had three lumens separated by submucosa within the same muscular layer, which extended fully through the length of the appendix. This case broadens the diversity of variations described in appendiceal triplications.

\section{BRAF (V600E) AND NRAS (Q61R/Q61L) MUTATION ANALYSIS USING IMMUNOHISTOCHEMISTRY: IMPLICATIONS FOR ROUTINE MUTATION TESTING OF MELANOMA SAMPLES}

Hojabr Kakavand $^{1,2}$, Trina Lum ${ }^{3}$, James S. Wilmott ${ }^{1,2}$, Elizabeth Smith $^{1,2}$, Sandra O'Toole ${ }^{1,3}$, Georgina V. Long ${ }^{1,2}$, Richard A. Scolyer ${ }^{1,2,3}$

${ }^{1}$ Sydney Medical School, the University of Sydney, Camperdown, ${ }^{2}$ Melanoma Institute Australia, North Sydney, and ${ }^{3}$ Tissue Pathology and Diagnostic Oncology, Royal Prince Alfred Hospital, Camperdown, NSW, Australia

Targeted therapies, such as BRAF and MEK inhibitors, have significantly improved the outcome of patients with metastatic melanoma. The appropriate administration of these therapies is dependent on the presence of mutations such as the BRAFV600E (prevalence 30-35\%), NRAS Q61R (10\%) and NRAS Q61L (5\%). There are various methods for mutation testing of samples each of which has varying sensitivities, costs and time allocations. The aim of this study was to assess the sensitivity and specificity of $B R A F$ and NRAS antibodies in a predefined cohort of patients with known mutation status. Three hundred and two metastatic melanoma samples with known mutation status using the Oncocarta panel (Sequenom) were included in this study. There were 92 patients with the BRAFV600E mutation, 28 with the BRAFV600K mutation, 50 with the NRASQ61R mutation, 22 with the NRASQ61L mutation, 20 with the NRASQ61K mutation and 90 that were wild type for both $B R A F$ and NRAS. Tissue microarrays were constructed and all samples were analysed using immunohistochemistry (IHC) for the BRAFV600E (VE1Spring Bioscience), NRASQ61R (SP174-Spring Bioscience) and NRASQ61L (26193-NewEast Biosciences) mutations. The staining was found to be homogeneous and cytoplasmic for all three antibodies. The sensitivity and specificity of each of the antibodies was analysed. The use of IHC is a fast, efficient and cost effective method to routinely screen patients for specific mutations. This will help facilitate the timely treatment of metastatic melanoma patients with targeted therapies.

\section{PRIMARY RETROPERITONEAL MUCINOUS CYSTADENOMA WITH BORDERLINE MALIGNANCY}

Laurne Kalinowski $^{1,3}$, Sally Byford ${ }^{2}$, Joanna Perry-Keene ${ }^{1}$ ${ }^{1}$ Department of Anatomical Pathology, ${ }^{2}$ Department of Obstetrics and Gynaecology, Nambour Base Hospital, Nambour, and ${ }^{3}$ Department of Pathology, University of Queensland, Brisbane, Qld, Australia

Primary retroperitoneal mucinous cystadenomas are rare with only seventeen cases previously reported in the literature with borderline features of malignancy. Primary retroperitoneal mucinous cystadenomas arise within the retroperitoneum without any connection to other organs and whilst predominantly occurring in women, cases are also described in men. We report a case in a 45-year-old woman who presented with an abdominal mass presumed ovarian in origin and benign on investigation. However, intra-operatively the mass was found to be retroperitoneal and histologically demonstrated borderline features. This case report includes a brief review of the literature examining primary retroperitoneal mucinous cystadenomas with borderline features and aims to highlight the 